\title{
In the View of Endothelial Microparticles: Novel Perspectives for Diagnostic and Pharmacological Management of Cardiovascular Risk during Diabetes Distress
}

\author{
Larissa Pernomian $\mathbb{D}^{1},{ }^{1}$ Jôsimar Dornelas Moreira, ${ }^{2}$ and Mayara Santos Gomes ${ }^{3}$ \\ ${ }^{1}$ Department of Biosciences Applied to Pharmacy, Faculty of Pharmaceutical Sciences from Ribeirão Preto, University of São Paulo, \\ Ribeirão Preto, SP, Brazil \\ ${ }^{2}$ Department of Clinical and Toxicological Analysis, Faculty of Pharmacy, Federal University of Minas Gerais, Belo Horizonte, \\ $M G$, Brazil \\ ${ }^{3}$ Department of Physics and Chemistry, Faculty of Pharmaceutical Sciences from Ribeirão Preto, University of São Paulo, \\ Ribeirão Preto, SP, Brazil \\ Correspondence should be addressed to Larissa Pernomian; l.pernomian@usp.br
}

Received 1 February 2018; Revised 27 March 2018; Accepted 26 April 2018; Published 13 May 2018

Academic Editor: Mark Yorek

Copyright ( 2018 Larissa Pernomian et al. This is an open access article distributed under the Creative Commons Attribution License, which permits unrestricted use, distribution, and reproduction in any medium, provided the original work is properly cited.

\begin{abstract}
Acute or chronic exposure to diabetes-related stressors triggers a specific psychological and behavior stress syndrome called diabetes distress, which underlies depressive symptoms in most diabetic patients. Distressed and/or depressive diabetic adults exhibit higher rates of cardiovascular mortality and morbidity, which have been correlated to macrovascular complications evoked by diabetic behavior stress. Recent experimental findings clearly point out that oxidative stress accounts for the vascular dysfunction initiated by the exposure to life stressors in diabetic conditions. Moreover, oxidative stress has been described as the main autocrine and paracrine mechanism of cardiovascular damage induced by endothelial microparticles (anuclear ectosomal microvesicles released from injured endothelial cells) in diabetic subjects. Such robust relationship between oxidative stress and cardiovascular diseases strongly suggests a critical role for endothelial microparticles as the primer messengers of the redox-dependent vascular dysfunction underlying diabetes distress. Here, we provide novel perspectives opened in the view of endothelial microparticles as promising diagnostic and pharmacotherapeutic biomarkers of cardiovascular risk in distressed diabetic patients.
\end{abstract}

\section{Introduction}

Diabetes distress has been described as a psychological and behavior stress syndrome of emotional symptoms that affect almost $50 \%$ of total diabetic patients [1-7]. Distressed diabetic patients exhibit higher rates of cardiovascular mortality and morbidity, which suggest a strong positive correlation between diabetes distress and cardiovascular risk of developing hypertension, dyslipidemia, atherosclerosis, myocardial infarction, stroke, and sudden death [8-15]. A few recent studies have shown that oxidative stress seems to be the key regulator of diabetes distress-induced cardiovascular diseases by impairing local anti-inflammatory nitrergic signaling
[3, 16-20]. Beyond the redox-mediated cardiovascular dysfunction proposed in diabetes distress, submicron vesicles shed from activated or quiescent endothelial cells, called endothelial microparticles (EMPs), have been pointed out as the main mediators of cardiovascular dysfunction underlying isolated diabetes by inducing proinflammatory pathways. In brief, EMP generation is increased by the typical oxidative stress induced in diabetic subjects affected by diabetes-related cardiovascular complications [21-25]. Taken together, these findings suggest that EMPs could represent a putative link between diabetes distress and the underlying redox-mediated cardiovascular dysfunction. Since there are no reports regarding the potential role assigned to EMPs as 
the downstream mediators of diabetes distress-related cardiovascular diseases, here we provide hypothetical mechanistic insights thereon by opening novel perspectives for the forthcoming use of EMPs as a diagnostic tool and pharmacological target for managing cardiovascular risk in distressed diabetic patients.

\section{Diabetes Distress: Can Stress Hurt?}

Considering that this question is not as simple as it seems to be, the more appropriate answer would be: it depends on how long stress lasts and how regulated the stress-based reactions are. If we understand psychological stress as an adaptive behavior resultant from transient neurological and hormonal responses induced for the sole purpose of ensuring survival [26], we would conclude that stress protects instead of hurts. However, if stress lasts for a long enough time and/or its reactions run on unregulated pathways, it can certainly hurt by triggering or contributing to the advance of a pathological environment [27]. In both cases, the outcomes of psychological stress arise from the hyperactivation of the hypothalamic-pituitary axis (HPA), whose tissue and systemic effects comprise extensive transcriptional and epigenetic changes [28].

In the view of diabetes, there are innumerous diseaserelated stressors that acutely or chronically activate HPA in an unregulated fashion, which leads to irregular peaks of serum corticosterone [29]. Overall, diabetes-related stressors comprise the following: significant emotional reactions to the diagnosis; requirement of self-management; blame and denial in dealing with the disease, the oppressive selfmanagement, and the social restrictions surrounding diabetic conditions; threats of diabetic complications; general diabetic depletion; and potential loss of function [1-3]. The irregular corticosterone peaks induced by diabetes-related stressors feedback their own behavior effects [30], leading to an expected stress reaction called diabetes distress, which rises as an affective experience involving wide emotional responses such as fears, worries, concerns, blame, and burden [2-7]. In addition to the behavior effects, the irregular corticosterone peaks induced by diabetes-related stressors also enhance diabetic insulin resistance and hyperglycemia, which contribute to the progression of diabetes [30]. For these reasons, diabetes distress is also correlated to a suboptimal glycemic control $[4,31,32]$.

Diabetes distress is a very common condition so that up to $45 \%$ of type 2 -diabetic patients experience its emotional symptoms [1-3]. This high incidence reduces the complacence of distressed diabetic patients for pharmacotherapy, diet, or physical exercises in $5-15 \%, 30 \%$, or $80 \%$, respectively [33]. For these reasons, these subjects exhibit higher rates of mortality correlated to all causes [34].

Although diabetes distress is a specific psychological and behavior stress syndrome that belongs to the diabetes spectrum, it has been described as a nonspecific indicator of clinical psychiatric comorbidities since it underlies major depression, elevated depressive symptoms, and subclinical depression that are widely developed by diabetic patients [2-7]. About $20-30 \%$ of distressed diabetic patients experience clinical depressive symptoms $[4,35]$ so that the prevalence of major depression is two- or three-fold higher in type 2- or type 1-diabetic patients than in the general population, respectively $[3,36]$. Both diabetes distress and major depression prognosis worsen when the two conditions coexist [37]: while HPA hyperactivity in distressed diabetic patients contributes to the progression of clinical depression, sympathomedular activation in depressive patients contributes to the insulin resistance during diabetes progression [38]. Taken together, these considerations support the importance of appropriately treating distressed diabetic patients in an attempt to avoid stress hurting. For these purposes, an effective glycemic control associated to behaviorcognitive approaches for improving diabetic prognosis and controlling the emotional stress and the eventual coexistent psychiatric symptoms has been considered one of the most effective choices [39].

\section{Diabetes Distress and Cardiovascular Risk: What Is in Common?}

There is much in common between diabetes distress and cardiovascular risk, starting with their prevalence as comorbidities. It is well documented that cardiovascular diseases represent the major cause of morbidity and mortality among diabetic patients, who exhibit a two-fold higher cardiovascular risk than the normoglycemic population [16, 40]. However, this higher incidence does not depend only on the traditional risk factors for cardiovascular diseases but it is strongly correlated to the behavior risk factors [40], pointing at diabetes distress as the major cause of cardiovascular complications underlying chronic hyperglycemia in stressed diabetic subjects [8].

Accordingly, distressed diabetic patients have a 1.69-fold higher risk for developing cardiovascular events [9], a 1.8-fold higher risk for developing cardiac ischemic diseases [10], and a 1.76-fold higher risk of cardiovascular mortality [9]. When diabetes distress is followed by clinical psychiatric disorders, cardiovascular risk gets a substantial increase of $39 \%$ [11] so that distressed depressive diabetic patients have a 2.4- to 3.5-fold higher risk of cardiovascular mortality [12]. Hypertension and macrovascular complications (myocardial infarction, stroke, and sudden death) are the most expressive cardiovascular events expressed in these patients, counting on a risk of 56\% [13] and 24-82\% $[14,15]$, respectively.

A marked positive correlation between diabetes distress and the main biomarkers of cardiovascular diseases (i.e., glycosylated hemoglobin (HbA1c), diastolic pressure, and low-density lipoprotein-cholesterol (LDL-c)) was recently confirmed in the most common cardiovascular events expressed in these patients [3, 16]. Accordingly, several studies have attempted to elucidate the mechanisms underlying the increased cardiovascular risk in diabetes distress. In this sense, recent studies have confirmed the critical role played by oxidative stress in exacerbating vascular diseases during experimental diabetes distress, which is induced by the behavior restraint stress model. Moreira et al. [17, 18] have showed that the contractile carotid hyperreactivity 
TABLE 1: Biomarkers of diabetes distress-related cardiovascular risk.

\begin{tabular}{|c|c|c|c|}
\hline Study & Biomarker & Cardiovascular disease & References \\
\hline \multirow{5}{*}{ DD-T2DM patients } & $\uparrow \mathrm{HbAlc}$ & Microvascular outcomes & Kreider [3] \\
\hline & $\uparrow$ Diastolic pressure & Macrovascular outcomes & Winchester et al. [16] \\
\hline & \multirow{3}{*}{$\uparrow \mathrm{LDL}-\mathrm{c}$} & Hypertension & \\
\hline & & Dyslipidemia & \\
\hline & & Atherosclerosis & \\
\hline \multirow{5}{*}{ AS-T1DM rats } & $\uparrow$ Vascular NADPH oxidase/Nox4 functionality & Carotid disease & \multirow{5}{*}{ Moreira et al. $[17,18]$} \\
\hline & $\uparrow$ Vascular Nox4-derived $\mathrm{H}_{2} \mathrm{O}_{2}$ levels & \multirow{4}{*}{ Stroke } & \\
\hline & $\uparrow$ Vascular iNOS functionality & & \\
\hline & $\uparrow$ Vascular iNOS-derived $\mathrm{ONOO}^{-}$levels & & \\
\hline & $\uparrow$ Vascular RAS functionality & & \\
\hline \multirow{5}{*}{ CS-DS/obese rats } & $\uparrow$ Cardiac NADPH oxidase functionality & & \multirow{5}{*}{ Matsuura et al. [20] } \\
\hline & $\uparrow$ Cardiac NADPH oxidase-derived $\mathrm{O}_{2}^{-}$levels & & \\
\hline & $\uparrow$ Cardiac RAS functionality & & \\
\hline & $\uparrow$ Cardiac macrophage infiltration & & \\
\hline & $\uparrow$ Cardiac inflammation & & \\
\hline
\end{tabular}

DD-T2DM patients (distressed depressive patients with type-2 diabetes mellitus); AS-T1DM rats (acutely stressed rats with type 1 diabetes mellitus); CS-DS/obese rats (chronically stressed rats with metabolic syndrome); HbA1C (glycosylated hemoglobin); LDL-c (low-density lipoprotein-cholesterol); $\mathrm{H}_{2} \mathrm{O}_{2}$ (hydrogen peroxide); iNOS (inducible nitric oxide synthase); $\mathrm{ONOO}^{-}$(peroxynitrite); RAS (renin-angiotensin system); $\mathrm{O}_{2}^{-}$(superoxide anion).

underlying cerebrovascular complication in stressed diabetic animals involves the upregulation of NADPH/Nox4 oxidasedriven generation of hydrogen peroxide $\left(\mathrm{H}_{2} \mathrm{O}_{2}\right)$ [17] and inducible nitric oxide synthase ( $i \mathrm{NOS})$-driven generation of peroxynitrite $\left(\mathrm{ONOO}^{-}\right)$[18]. The authors have demonstrated that the increased generation of $\mathrm{H}_{2} \mathrm{O}_{2}$ and $\mathrm{ONOO}^{-}$ is induced by the acute exposure of rats to restraint stress, which increases carotid resistance by enhancing the local preexistent diabetes-related angiotensinergic hyperreactivity [19] due to the contractile effects evoked by the referred reactive oxygen species (ROS) $[17,18]$. In agreement with these findings, Matsuura et al. [20] showed that the chronic exposure of animals with metabolic syndrome (DahlS.ZLepr $^{\mathrm{fa}} /$ Lepr $^{\mathrm{fa}} /$ obese rats) to restraint stress exacerbates the preexisting hypertension, ventricular hypertrophy fibrosis, and diastolic dysfunction by cardiovascular oxidative stressand proinflammatory-dependent mechanisms and hyperactivation of the local renin-angiotensin system (RAS). The clinical and experimental findings regarding the biomarkers of diabetes distress-related cardiovascular risk are summarized in Table 1.

The experimental correlations assigned to reninangiotensin system (RAS) activation and redox signaling suggest vascular dysfunction as the putative key mechanism underlying the increased cardiovascular risk during diabetes distress $[3,16,17,20]$. Indeed, angiotensinergic oxidative stress has been described as a critical mechanism for inducing general vascular dysfunction under both stressful [41-44] and hyperglycemic [19] stimuli. In turn, vascular dysfunction underlies all of the diabetic cardiovascular complications during their early preclinical stages [45]. Thus, the identification of integrative molecular players of redoxdependent vascular dysfunction provides a novel pathophysiological understanding of cardiovascular risk in diabetes distress and novel insights for selectively targeting vascular competence for diagnosis, prognosis, and therapy purposes.

\section{Cardiovascular Risk in Diabetes Distress and Endothelial Microparticles: Could They Be Linked?}

When taking oxidative stress into account, cardiovascular risk in diabetes distress can certainly be linked to endothelial microparticles (EMPs), which have been described as noninvasive surrogate biomarkers of redox-induced vascular injury and dysfunction correlated with poor clinical outcomes in cardiovascular diseases [45]. In brief, EMPs are submicron vesicles ranging from 0.1 to $1.0 \mu \mathrm{m}$ in diameter, shed from the blebbing of plasma membrane of activated or quiescent endothelial cells during prooxidant events that lead to a direct effect of ROS on the vasculature such as shear stress-induced blood pressure disorders (which induces ROS generation upon NADPH oxidase activation), the agonist activation of receptors attached to redox signaling (such as angiotensin $\mathrm{AT}_{1}$, endothelin $\mathrm{ET}_{\mathrm{A}}$, endothelin $\mathrm{ET}_{\mathrm{B}}$, and TNF $\alpha$-I receptors), the redox-induced cell apoptosis, and/or the oxidative stress-mediated mitochondrial dysfunction [21, 46-48]. As autocrine and paracrine mediators, EMPs elicit oxidative stress in endothelial cells by increasing the local ROS generation and triggering underlying redoxproinflammatory mechanisms that (1) downregulate nitric oxide (NO) release by inducing the uncoupling of endothelial nitric oxide synthase ( $e$ NOS) and (2) disrupt the endothelium competence on nitrergic anti-inflammatory signaling [49]. This effect may be due to the expression of functionally active NADPH oxidase subunits $\left(p 22^{\text {phox }}, p 38^{\text {phox }}, p 47^{\text {phox }}\right.$, $p 67^{\text {phox }}$, Nox1, Nox2, and Nox4) in EMPs [50]. 
Circulating EMPs represent the minority of total microparticles in healthy conditions but their levels are substantially increased by the oxidative stress-induced lipid peroxidation in endothelial cells during diabetes or cardiovascular diseases [21-25]. Moreover, diabetic cardiovascular complications increase the circulating levels of EMPs when compared to isolated diabetes or cardiovascular diseases $[51,52]$. Interestingly, diabetes seems to increase EMPs' competence in inducing vascular dysfunction. Jansen et al. [53] recently showed that the redox-dependent vascular inflammation in atherosclerotic lesions (i.e., macrophage infiltration and adhesion protein expression) is higher when induced by EMPs derived from endothelial cells exposed to diabetes-like hyperglycemic conditions. Accordingly, although high glucose levels did not change EMPs' morphology (i.e., size and antigen composition), the hyperglycemia increases the functionality of EMPs in activating $\mathrm{NADPH}$ oxidase $/ p 38^{\text {phox }}$-mediated signaling when compared to EMPs formed in a normoglycemic environment [53]. The clinical significance of such redox/proinflammatory potential assigned to diabetic EMPs lies in their prognostic value for coronary artery disease in diabetes due to the positive correlation between the high circulating EMP levels and the severity of vascular dysfunction in diabetic patients $[23,54,55]$. Taken together with the well-described remarkable increase in vascular inflammation, vascular ROS generation, and vascular NAPH oxidase complex expression and functionality in animal models of diabetes distress [17-20], the stronger competence of diabetic EMPs in inducing oxidative vascular injury and dysfunction points at EMPs as feasible integrative molecular players of the increased cardiovascular risk in diabetes distress $[8-16,40]$. Such hypothetical mechanistic insights are summarized in Figure 1. Therefore, selectively targeting EMPs may represent a promising perspective for effective diagnostic, prognostic, and therapeutic purposes aimed at the cardiovascular risk in distressed diabetic patients.

\section{In the view of Endothelial Microparticles: Novel Insights for Cardiovascular Risk Management in Diabetes Distress!}

The recent findings described here concerning the oxidative potential assigned to EMPs in inducing diabetic cardiovascular complications open real perspectives for managing cardiovascular risk during diabetes distress by considering EMPs as diagnostic key hallmarks and pharmacological targets. For diagnostic purposes, the circulating levels of EMPs can be quantified by polychromatic flow cytometry as the simplest method that provides valuable information regarding the antigen composition and size criteria $[56,57]$. In these cases, platelet-poor plasma must be obtained by serial centrifugation or ultracentrifugation of freshly drawn whole blood samples, since freezing or storage approaches disrupt the microparticles' integrity. Also, the blood samples must be pretreated with calcium $\left(\mathrm{Ca}^{2+}\right)$-chelator anticoagulants (e.g., EDTA or sodium citrate) for avoiding microparticle aggregation. Once prepared, the platelet-poor plasma is

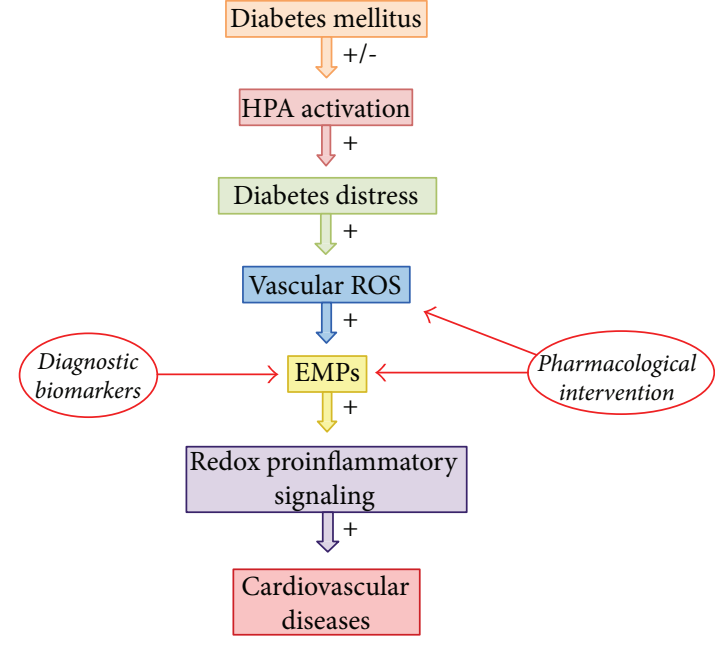

Figure 1: Hypothetical mechanistic insights regarding the potential role played by endothelial microparticles as key downstream mediators of diabetes distress-related cardiovascular diseases. The irregular activation of HPA in diabetic patients leads to the psychological and behavior stress syndrome called diabetes distress, which induces vascular oxidative stress by enhancing the functionality of the renin-angiotensin system and NADPH oxidase; the resultant ROS activate EMP generation upon redox-mediated quiescence of endothelial cells; finally, EMPs trigger redox proinflammatory signaling in autocrine and paracrine mechanisms that underlies cardiovascular diseases positively correlated with diabetes distress. Such putative role assigned to EMPs opens novel perspectives in using the generation of EMPs as a biomarker and a target for diagnostic and pharmacological intervention aimed at the managing of diabetes distress-related cardiovascular risk, respectively. HPA (hypothalamic-pituitary axis); ROS (reactive oxygen species); EMPs (endothelial microparticles).

labeled with fluorescent monoclonal antibodies for staining specific surface antigens expressed on endothelial cells such as CD31 and CD54, and the cytometric analysis is performed by applying forward and side light scatter with calibration beads of a known diameter [58].

A few recent studies have shown that some specific pharmacological interventions are able to promote protective effects by significantly reducing circulating levels of EMPs in diabetic patients affected by cardiovascular diseases including hypertension. For instance, the $\mathrm{Ca}^{2+}$ blockers nifedipine and benifipine, combined or not with the angiotensin $\mathrm{AT}_{1}$ antagonist losartan, were able to reduce EMP formation in hypertensive diabetic patients by antioxidant mechanisms that inhibited lipid peroxidation in endothelial cells rather than hypotensive effects [59-61]. Similarly, statins such as simvastatin and fluvastatin, as well as vitamin $\mathrm{C}$ and losartan, reduced the circulating levels of EMPs in hypertensive hyperlipidemic diabetic patients affected by advanced atherosclerotic plaques [22, 60, 62-64]. Interestingly, the therapeutic effects of these drugs on EMP circulating levels result from pharmacological actions that interfere with the mechanisms of EMP formation. Indeed, it would not be feasible to selectively target EMPs with specific antibodies or scavengers since other microparticles stemmed from different parental 
cells share some of the surface antigens expressed by EMPs including CD31 and CD54 [65]. Currently, these mechanisms are summarized as the shedding of activated or quiescent endothelial cell plasma membrane blebbing, which can result from the increase of intracellular $\mathrm{Ca}^{2+}$ or ROS levels induced by shear stress, agonist receptor activation (including the activation of angiotensin $\mathrm{AT}_{1}$, endothelin $\mathrm{ET}_{\mathrm{A}}$, endothelin $\mathrm{ET}_{\mathrm{B}}$, and TNF $\alpha$-I receptors), apoptosis, or oxidative stress-mediated mitochondrial dysfunction [21, 46-48, 66-68]. However, recent studies have shown that lipid-lowering therapies inhibit EMP formation by an unknown mechanism $[69,70]$ that putatively interferes with the Rho-kinase pathway [63]. Such information highlights the importance of accurately identifying all the pathophysiological mechanisms of EMP formation so that they can be defined as pharmacological targets for controlling cardiovascular risk in distressed diabetic patients.

Although there are no reports concerning the direct effects of selective serotonin reuptake inhibitors (IRS) on circulating EMP levels from depressive distressed diabetic patients [71], experimental findings have shown that escitalopram and paroxetine prevent vascular dysfunction in chronically stressed or diabetic rats by cardiac and endothelial antioxidant mechanisms [72-74]. Taken together, these findings suggest a novel therapeutic role for IRS as protective agents on the cardiovascular system by putatively inhibiting the redox-dependent formation of EMPs during stressful and/or diabetic conditions. The confirmation of this hypothesis would then make IRS an important pharmacological tool as reliable as $\mathrm{Ca}^{2+}$ blockers, statins, $\mathrm{AT}_{1}$ antagonists, or antioxidants for managing cardiovascular risk in diabetes distress.

\section{Conflicts of Interest}

The authors declare that there is no conflict of interest regarding the publication of this article.

\section{Acknowledgments}

The authors thank Professor Cristina Ribeiro de Barros Cardoso for her scientific support. This work was supported by the Coordination for the Improvement of Higher Education Personnel (CAPES), by the National Postdoctorate Program of CAPES (PNPD/CAPES), and by the São Paulo Research Foundation (FAPESP, Brazil, Grant nos. 2014/ 17740-0, 2013/08216-2, 2012/00640-7, 2012/09019-3, and 2009/01005-0).

\section{References}

[1] L. Fisher, J. C. N. Chan, H. Nan, N. Sartorius, and B. Oldenburg, "Co-occurrence of diabetes and depression: conceptual considerations for an emerging global health challenge," Journal of Affective Disorders, vol. 142, pp. S56S66, 2012.

[2] L. Fisher, J. S. Gonzalez, and W. H. Polonsky, "The confusing tale of depression and distress in patients with diabetes: a call for greater clarity and precision," Diabetic Medicine, vol. 31, no. 7, pp. 764-772, 2014.
[3] K. E. Kreider, "Diabetes distress or major depressive disorder? A practical approach to diagnosing and treating psychological comorbidities of diabetes," Diabetes Therapy, vol. 8, no. 1, pp. 1-7, 2017.

[4] L. Fisher, J. T. Mullan, P. Arean, R. E. Glasgow, D. Hessler, and U. Masharani, "Diabetes distress but not clinical depression or depressive symptoms is associated with glycemic control in both cross-sectional and longitudinal analyses," Diabetes Care, vol. 33, no. 1, pp. 23-28, 2010.

[5] J. S. Gonzalez, L. Fisher, and W. H. Polonsky, "Depression in diabetes: have we been missing something important?," Diabetes Care, vol. 34, no. 1, pp. 236-239, 2011.

[6] S. A. Esbitt, M. L. Tanenbaum, and J. S. Gonzalez, "Disentangling clinical depression from diabetes-specific distress: making sense of the mess we've made," in Screening for Depression and Other Psychological Problems in Diabetes, C. E. Lloyd, F. Pouwer, and N. Hermans, Eds., Springer, London, 2013.

[7] L. Gask, W. Macdonald, and P. Bower, "What is the relationship between diabetes and depression? A qualitative metasynthesis of patient experience of co-morbidity," Chronic Illness, vol. 7, no. 3, pp. 239-252, 2011.

[8] K. Semenkovich, M. E. Brown, D. M. Svrakic, and P. J. Lustman, "Depression in type 2 diabetes mellitus: prevalence, impact, and treatment," Drugs, vol. 75, no. 6, pp. 577-587, 2015.

[9] E.-M. Dalsgaard, M. Vestergaard, M. V. Skriver et al., "Psychological distress, cardiovascular complications and mortality among people with screen-detected type 2 diabetes: followup of the ADDITION-Denmark trial," Diabetologia, vol. 57, no. 4, pp. 710-717, 2014.

[10] M. C. Adriaanse, F. Pouwer, J. M. Dekker et al., "Diabetesrelated symptom distress in association with glucose metabolism and comorbidity," Diabetes Care, vol. 31, no. 12, pp. 2268-2270, 2008.

[11] R. J. Anderson, A. B. Grigsby, K. E. Freedland et al., “Anxiety and poor glycemic control: a meta-analytic review of the literature," The International Journal of Psychiatry in Medicine, vol. 32, no. 3, pp. 235-247, 2002.

[12] L. Egede, P. J. Nietert, and D. Zheng, "Depression and all-cause and coronary heart disease mortality among adults with and without diabetes," Diabetes Care, vol. 28, no. 6, pp. 13391345, 2005.

[13] A. K. Khuwaja, S. Lalani, R. Dhanani, I. S. Azam, G. Rafique, and F. White, "Anxiety and depression among outpatients with type 2 diabetes: a multi-centre study of prevalence and associated factors," Diabetology \& Metabolic Syndrome, vol. 2, no. 1, pp. 72-77, 2010.

[14] E. H. B. Lin, C. M. Rutter, W. Katon et al., "Depression and advanced complications of diabetes: a prospective cohort study," Diabetes Care, vol. 33, no. 2, pp. 264-269, 2010.

[15] J. F. Scherrer, L. D. Garfield, T. Chrusciel et al., "Increased risk of myocardial infarction in depressed patients with type 2 diabetes," Diabetes Care, vol. 34, no. 8, pp. 1729-1734, 2011.

[16] R. J. Winchester, J. S. Williams, T. E. Wolfman, and L. E. Egede, "Depressive symptoms, serious psychological distress, diabetes distress and cardiovascular risk factor control in patients with type 2 diabetes," Journal of Diabetes and its Complications, vol. 30, no. 2, pp. 312-317, 2016.

[17] J. D. Moreira, L. Pernomian, M. S. Gomes et al., "Acute restraint stress increases carotid reactivity in type-I diabetic 
rats by enhancing Nox4/NADPH oxidase functionality," European Journal of Pharmacology, vol. 765, pp. 503-516, 2015.

[18] J. D. Moreira, L. Pernomian, M. S. Gomes et al., "Enhanced nitric oxide generation from nitric oxide synthases as the cause of increased peroxynitrite formation during acute restraint stress: effects on carotid responsiveness to angiotensinergic stimuli in type-1 diabetic rats," European Journal of Pharmacology, vol. 783, pp. 11-22, 2016.

[19] L. Pernomian, M. S. Gomes, C. B. A. Restini, L. N. Z. Ramalho, C. R. Tirapelli, and A. M. de Oliveira, "The role of reactive oxygen species in the modulation of the contraction induced by angiotensin II in carotid artery from diabetic rat," European Journal of Pharmacology, vol. 678, no. 1-3, pp. 15-25, 2012.

[20] N. Matsuura, K. Nagasawa, Y. Minagawa et al., "Restraint stress exacerbates cardiac and adipose tissue pathology via $\beta$-adrenergic signaling in rats with metabolic syndrome," American Journal of Physiology Heart and Circulatory Physiology, vol. 308, no. 10, pp. H1275-H1286, 2015.

[21] A. S. Leroyer, A. Tedgui, and C. M. Boulanger, "Microparticles and type 2 diabetes," Diabetes \& Metabolism, vol. 34, pp. 2732,2008

[22] O. Morel, L. Jesel, B. Hugel et al., "Protective effects of vitamin $\mathrm{C}$ on endothelium damage and platelet activation during myocardial infarction in patients with sustained generation of circulating microparticles," Journal of Thrombosis and Haemostasis, vol. 1, no. 1, pp. 171-177, 2003.

[23] H. Koga, S. Sugiyama, K. Kugiyama et al., "Elevated levels of VE-cadherin-positive endothelial microparticles in patients with type 2 diabetes mellitus and coronary artery disease," Journal of the American College of Cardiology, vol. 45, no. 10, pp. 1622-1630, 2005.

[24] F. Sabatier, P. Darmon, B. Hugel et al., “Type 1 and type 2 diabetic patients display different patterns of cellular microparticles," Diabetes, vol. 51, no. 9, pp. 2840-2845, 2002.

[25] B. Feng, Y. Chen, Y. Luo, M. Chen, X. Li, and Y. Ni, "Circulating level of microparticles and their correlation with arterial elasticity and endothelium-dependent dilation in patients with type 2 diabetes mellitus," Atherosclerosis, vol. 208, no. 1, pp. 264-269, 2010.

[26] A. S. Zannas and G. P. Chrousos, "Epigenetic programming by stress and glucocorticoids along the human lifespan," Molecular Psychiatry, vol. 22, no. 5, pp. 640-646, 2017.

[27] S. Cohen, P. J. Gianaros, and S. B. Manuck, "A stage model of stress and disease," Perspectives on Psychological Science, vol. 11, no. 4, pp. 456-463, 2016.

[28] M. van Bodegom, J. R. Homberg, and M. J. A. G. Henckens, "Modulation of the hypothalamic-pituitary-adrenal axis by early life stress exposure," Frontiers in Cellular Neuroscience, vol. 11, p. 87, 2017.

[29] E. M. Kyritsi, G. Koltsida, I. Farakla et al., "Psychological vulnerability to stress in carriers of congenital adrenal hyperplasia due to 21-hydroxylase deficiency," Hormones, vol. 16, no. 1 , pp. $42-53,2017$.

[30] J. D. Shen, Y. Wei, Y. J. Li, J. Y. Qiao, and Y. C. Li, "Curcumin reverses the depressive-like behavior and insulin resistance induced by chronic mild stress," Metabolic Brain Disease, vol. 32, no. 4, pp. 1163-1172, 2017.

[31] L. Fisher, R. E. Glasgow, and L. A. Strycker, "The relationship between diabetes distress and clinical depression with glycemic control among patients with type 2 diabetes," Diabetes Care, vol. 33, no. 5, pp. 1034-1036, 2010.
[32] L. Fisher, M. M. Skaff, J. T. Mullan et al., "Clinical depression versus distress among patients with type 2 diabetes: not just a question of semantics," Diabetes Care, vol. 30, no. 3, pp. 542-548, 2007.

[33] H. Roohafza, A. Kabir, M. Sadeghi et al., "Stress as a risk factor for noncompliance with treatment regimens in patients with diabetes and hypertension," ARYA Atheroscler, vol. 12, no. 4, pp. 166-171, 2016.

[34] F. E. van Dooren, G. Nefs, M. T. Schram, F. R. J. Verhey, J. Denollet, and F. Pouwer, "Depression and risk of mortality in people with diabetes mellitus: a systematic review and meta-analysis," PLoS One, vol. 8, no. 3, article e57058, 2013.

[35] M. L. Tanebaum and J. S. Gonzalez, "The influence of diabetes on a clinician-rated assessment of depression in adults with type 1 diabetes," The Diabetes Educator, vol. 38, no. 5, pp. 695-704, 2012.

[36] T. Roy and C. E. Lloyd, "Epidemiology of depression and diabetes: a systematic review," Journal of Affective Disorders, vol. 142, pp. S8-S21, 2012.

[37] C. E. Lloyd, G. Pambianco, and T. J. Orchard, "Does diabetesrelated distress explain the presence of depressive symptoms and/or poor self-care in individuals with type 1 diabetes?," Diabetic Medicine, vol. 27, no. 2, pp. 234-237, 2010.

[38] J. K. Rustad, D. L. Musselman, and C. B. Nemeroff, "The relationship of depression and diabetes: pathophysiological and treatment implications," Psychoneuroendocrinology, vol. 36, no. 9, pp. 1276-1286, 2011.

[39] S. A. Safren, J. S. Gonzalez, D. J. Wexler et al., “A randomized controlled trial of cognitive behavioral therapy for adherence and depression (CBT-AD) in patients with uncontrolled type 2 diabetes," Diabetes Care, vol. 37, no. 3, pp. 625-633, 2014.

[40] R. A. Hackett and A. Steptoe, "Psychosocial factors in diabetes and cardiovascular risk," Current Cardiology Reports, vol. 18, no. 10, p. 95, 2016

[41] I. M. Chung, Y. M. Kim, M. H. Yoo, M. K. Shin, C. K. Kim, and S. H. Suh, "Immobilization stress induces endothelial dysfunction by oxidative stress via the activation of the angiotensin II/ its type I receptor pathway," Atherosclerosis, vol. 213, no. 1, pp. 109-114, 2010.

[42] M. Groeschel and B. Braam, "Connecting chronic and recurrent stress to vascular dysfunction: no relaxed role for the renin-angiotensin system," American Journal of Physiology Renal Physiology, vol. 300, no. 1, pp. F1-F10, 2011.

[43] W. Costa-Ferreira, J. O. Vieira, J. Almeida, L. Gomes-de-Souza, and C. C. Crestani, "Involvement of type 1 angiontensin II receptor $\left(\mathrm{AT}_{1}\right)$ in cardiovascular changes induced by chronic emotional stress: comparison between homotypic and heterotypic stressors," Frontiers in Pharmacology, vol. 7, p. 262, 2016.

[44] H. Côco, L. Pernomian, P. C. Pereira et al., "Chronic restraint stress increases angiotensin II potency in the rat carotid: role of cyclooxygenases and reactive oxygen species," Journal of Pharmacy and Pharmacology, vol. 69, no. 1, pp. 52-65, 2017.

[45] F. Sabatier, L. Camoin-Jau, F. Anfosso, J. Sampol, and F. Dignat-George, "Circulating endothelial cells, microparticles and progenitors: key players towards the definition of vascular competence," Journal of Cellular and Molecular Medicine, vol. 13, no. 3, pp. 454-471, 2009.

[46] F. Santilli, M. Marchisio, P. Lanuti, A. Boccatonda, S. Miscia, and G. Davì, "Microparticles as new markers of cardiovascular risk in diabetes and beyond," Thrombosis and Haemostasis, vol. 116, no. 2, pp. 220-234, 2016. 
[47] P. Puddu, G. M. Puddu, E. Cravero, S. Muscari, and A. Muscari, "The involvement of circulating microparticles in inflammation, coagulation and cardiovascular diseases," Canadian Journal of Cardiology, vol. 26, pp. e140-e145, 2010.

[48] S. Chan, Q. Lian, M. P. Chen et al., "Deferiprone inhibits iron overload-induced tissue factor bearing endothelial microparticle generation by inhibition oxidative stress induced mitochondrial injury, and apoptosis," Toxicology and Applied Pharmacology, vol. 338, pp. 148-158, 2018.

[49] M. E. Tushuizen, R. Nieuwland, C. Rustemeijer et al., "Elevated endothelial microparticles following consecutive meals are associated with vascular endothelial dysfunction in type 2 diabetes," Diabetes Care, vol. 30, no. 3, pp. 728-730, 2007.

[50] D. Burger, M. Turner, M. N. Munkonda, and R. M. Touyz, "Endothelial microparticle-derived reactive oxygen species: role in endothelial signaling and vascular function," Oxidative Medicine and Cellular Longevity, vol. 2016, Article ID 5047954, 10 pages, 2016.

[51] A. S. Leroyer, H. Isobe, G. Lesèche et al., "Cellular origins and thrombogenic activity of microparticles isolated from human atherosclerotic plaques," Journal of the American College of Cardiology, vol. 49, no. 7, pp. 772-777, 2007.

[52] K. Esposito, M. Ciotola, F. Giugliano et al., "Endothelial microparticles correlate with erectile dysfunction in diabetic men," International Journal of Impotence Research, vol. 19, no. 2, pp. 161-166, 2007.

[53] F. Jansen, X. Yang, B. S. Franklin et al., "High glucose condition increases NADPH oxidase activity in endothelial microparticles that promote vascular inflammation," Cardiovascular Research, vol. 98, no. 1, pp. 94-106, 2013.

[54] N. Amabile, A. P. Guérin, A. Leroyer et al., "Circulating endothelial microparticles are associated with vascular dysfunction in patients with end-stage renal failure," Journal of the American Society of Nephrology, vol. 16, no. 11, pp. 3381-3388, 2005.

[55] N. Werner, S. Wassmann, P. Ahlers, S. Kosiol, and G. Nickenig, "Circulating $\mathrm{CD}_{3} 1^{+} /$annexin $\mathrm{V}^{+}$apoptotic microparticles correlate with coronary endothelial function in patients with coronary artery disease," Arteriosclerosis, Thrombosis, and Vascular Biology, vol. 26, no. 1, pp. 112-116, 2006.

[56] F. Deng, S. Wang, and L. Zhang, "Endothelial microparticles act as novel diagnostic and therapeutic biomarkers of diabetes and its complications: a literature review," BioMed Research International, vol. 2016, Article ID 9802026, 8 pages, 2016.

[57] P. Lanuti, F. Santilli, M. Marchisio et al., "A novel flow cytometric approach to distinguish circulating endothelial cells from endothelial microparticles: relevance for the evaluation of endothelial dysfunction," Journal of Immunological Methods, vol. 380, no. 1-2, pp. 16-22, 2012.

[58] S. Nomura, "Microparticle and atherothrombotic diseases," Journal of Atherosclerosis and Thrombosis, vol. 23, no. 1, pp. 1-9, 2016.

[59] S. Nomura, N. Inami, Y. Kimura et al., "Effect of nifedipine on adiponectin in hypertensive patients with type 2 diabetes mellitus," Journal of Human Hypertension, vol. 21, no. 1, pp. 38-44, 2007.

[60] S. Nomura, A. Shouzu, S. Omoto, M. Nishikawa, S. Fukuhara, and T. Iwasaka, "Losartan and simvastatin inhibit platelet activation in hypertensive patients," Journal of Thrombosis and Thrombolysis, vol. 18, no. 3, pp. 177-185, 2004.

[61] S. Nomura, A. Shouzu, S. Omoto, M. Nishikawa, and T. Iwasaka, "Benidipine improves oxidized LDL-dependent monocyte and endothelial dysfunction in hypertensive patients with type 2 diabetes mellitus," Journal of Human Hypertension, vol. 19, no. 7, pp. 551-557, 2005.

[62] S. Nomura, A. Shouzu, S. Omoto et al., "Effects of eicosapentaenoic acid on endothelial cell-derived microparticles, angiopoietins and adiponectin in patients with type 2 diabetes," Journal of Atherosclerosis and Thrombosis, vol. 16, no. 2, pp. 83-90, 2009.

[63] A. F. Tramontano, J. O’Leary, A. D. Black, R. Muniyappa, M. V. Cutaia, and N. el-Sherif, "Statin decreases endothelial microparticle release from human coronary artery endothelial cells: implication for the Rho-kinase pathway," Biochemical and Biophysical Research Communications, vol. 320, no. 1, pp. 34-38, 2004.

[64] O. Morel, F. Toti, B. Hugel et al., "Procoagulant microparticles: disrupting the vascular homeostasis equation?," Arteriosclerosis, Thrombosis, and Vascular Biology, vol. 26, no. 12, pp. 2594-2604, 2006.

[65] C. N. França, M. C. Izar, J. B. Amaral, D. M. Tegani, and F. A. Fonseca, "Microparticles as potential biomarkers of cardiovascular disease," Arquivos Brasileiros de Cardiologia, vol. 104, no. 2, pp. 169-174, 2015.

[66] S. Yang, Q. Zhong, Z. Qiu et al., “Angiotensin II receptor type 1 autoantibodies promote endothelial microparticles formation through activating p38 MAPK pathway," Journal of Hypertension, vol. 32, no. 4, pp. 762-770, 2014.

[67] M. Lichtenayer, B. Goebel, V. Paar et al., "Acute effects of moderate altitude on biomarkers of cardiovascular inflammation and endothelial function and their differential modulation by dual endothelin receptor blockade," Clinical Hemorheology and Microcirculation, vol. 67, no. 1, pp. 101-113, 2017.

[68] S. K. Lee, S. H. Yang, I. Kwon, O. H. Lee, and J. H. Heo, "Role of tumour necrosis factor receptor- 1 and nuclear factor- $\kappa \mathrm{B}$ in production of TNF- $\alpha$-induced pro-inflammatory microparticles in endothelial cells," Thrombosis and Haemostasis, vol. 112, no. 2, pp. 580-588, 2014.

[69] R. Suades, T. Padró, R. Alonso, P. Mata, and L. Badimon, "Lipid-lowering therapy with statins reduces microparticle shedding from endothelium, platelets and inflammatory cells," Thrombosis and Haemostasis, vol. 110, no. 2, pp. 366-377, 2013.

[70] F. Mobarrez, N. Egberg, J. Antovic, A. Bröijersen, G. Jörneskog, and H. Wallén, "Release of endothelial microparticles in vivo during atorvastatin treatment; a randomized double-blind placebo-controlled study," Thrombosis Research, vol. 129, no. 1, pp. 95-97, 2012.

[71] S. Roopan and E. R. Larsen, "Use of antidepressants in patients with depression and comorbid diabetes mellitus: a systematic review," Acta Neuropsychiatrica, vol. 29, no. 3, pp. 127-139, 2017.

[72] V. V. Matchkov, V. V. Kravtsova, O. Wiborg, C. Aalkjaer, and E. V. Bouzinova, "Chronic selective serotonin reuptake inhibition modulates endothelial dysfunction and oxidative state in rat chronic mild stress model of depression," American Journal of Physiology Regulatory, Integrative and Comparative Physiology, vol. 309, no. 8, pp. R814-R823, 2015.

[73] D. Gerö, P. Szoleczky, K. Suzuki et al., "Cell-based screening identifies paroxetine as an inhibitor of diabetic endothelial dysfunction," Diabetes, vol. 62, no. 3, pp. 953-964, 2013.

[74] S. B. Wheatcroft, "Teaching an old drug new tricks: can paroxetine ease the burden of cardiovascular disease in diabetes?," Diabetes, vol. 62, no. 3, pp. 698-700, 2013. 


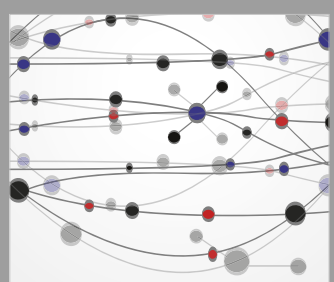

The Scientific World Journal
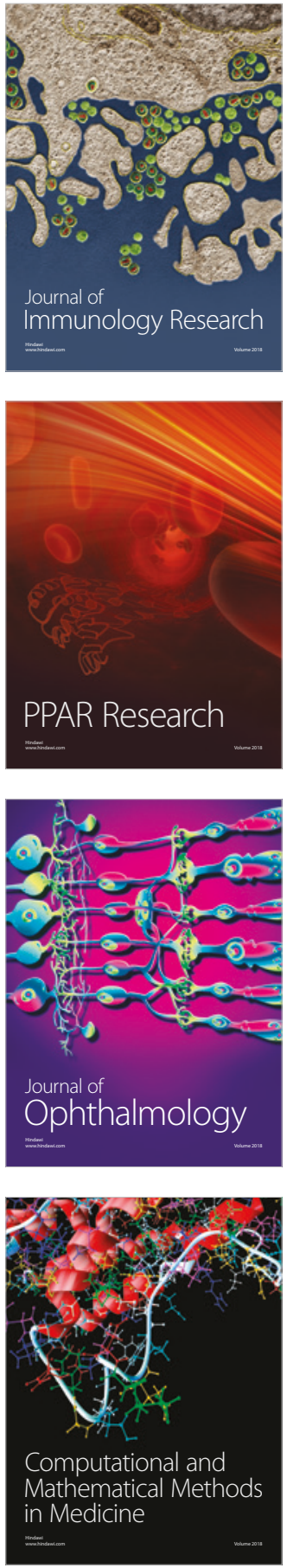

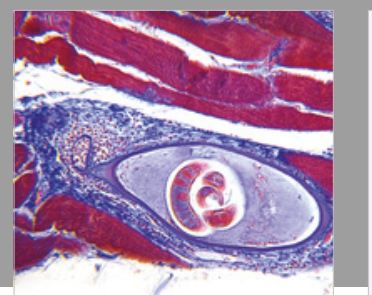

Gastroenterology Research and Practice

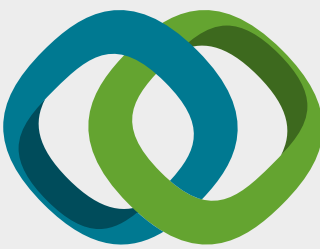

\section{Hindawi}

Submit your manuscripts at

www.hindawi.com
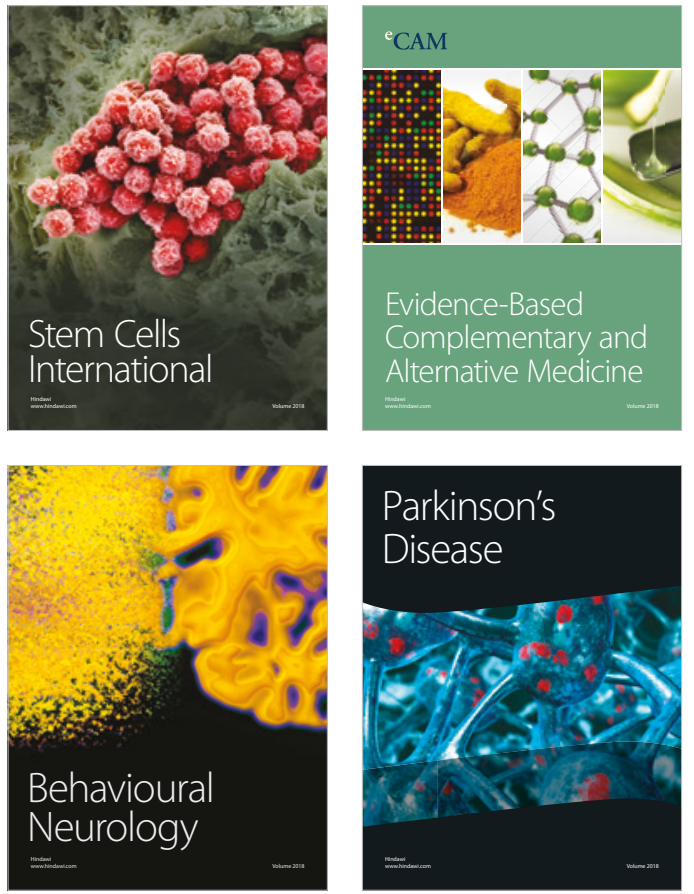

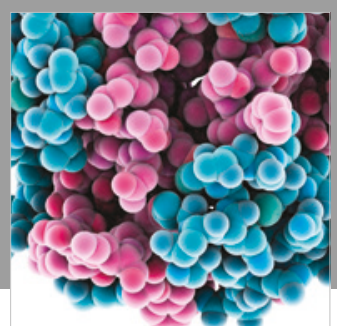

ournal of

Diabetes Research

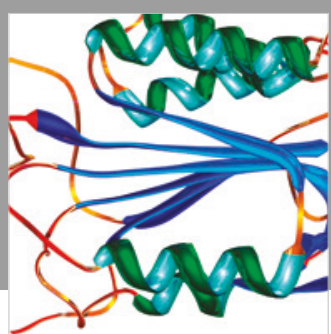

Disease Markers
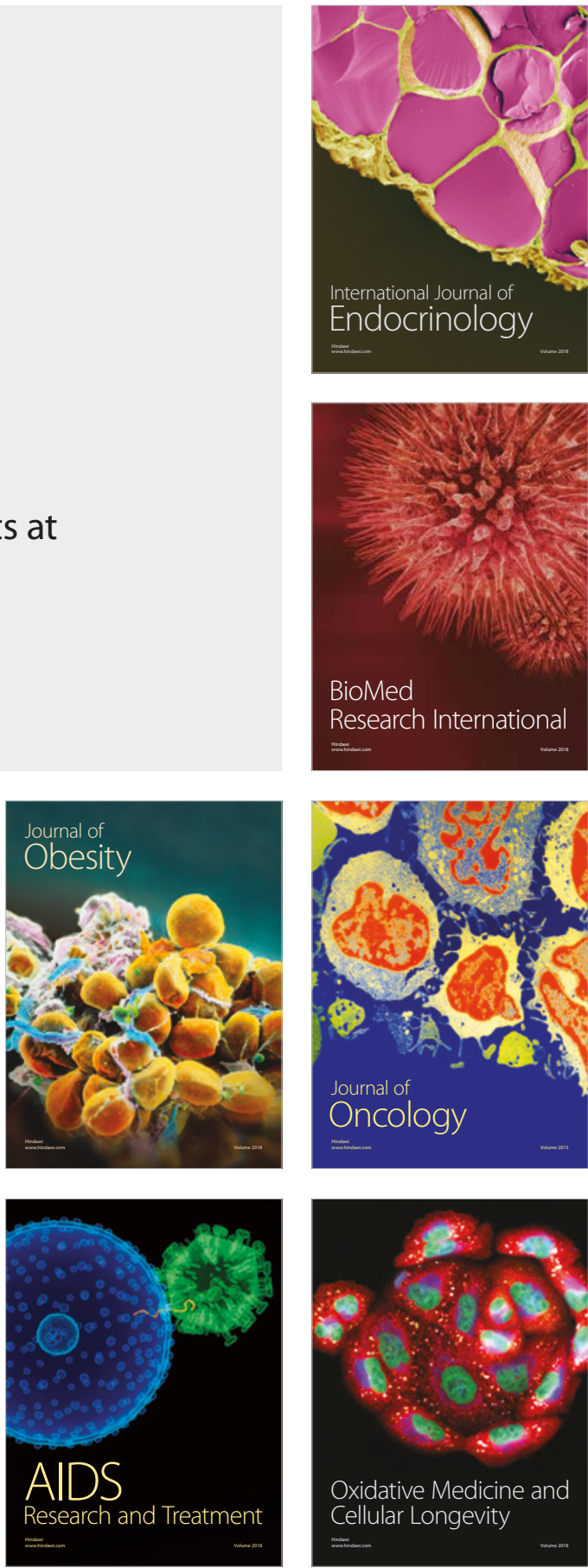